\title{
An Apéry-like difference equation for Catalan's constant
}

\author{
W. Zudilin \\ Moscow Lomonosov State University \\ Department of Mechanics and Mathematics \\ Vorobiovy Gory, GSP-2, Moscow 119992 RUSSIA \\ URL: http://wain.mi.ras.ru/index.html \\ E-mail address: wadim@ips.ras.ru
}

Submitted: Jan 18, 2002; Accepted: Mar 31, 2003; Published: Apr 23, 2003

MR Subject Classifications: Primary 11J70, 11Y60, 33F10;

Secondary 11B37, 11B65, 11M06, 33C20, 33C60, 39A05.

\begin{abstract}
Applying Zeilberger's algorithm of creative telescoping to a family of certain very-well-poised hypergeometric series involving linear forms in Catalan's constant with rational coefficients, we obtain a second-order difference equation for these forms and their coefficients. As a consequence we derive a new way of fast calculation of Catalan's constant as well as a new continued-fraction expansion for it. Similar arguments are put forward to deduce a second-order difference equation and a new continued fraction for $\zeta(4)=\pi^{4} / 90$.
\end{abstract}

\section{Introduction}

One of the most crucial and quite mysterious ingredients in Apéry's proof [1], [8] of the irrationality of $\zeta(2)$ and $\zeta(3)$ is the existence of the difference equations

$$
\begin{gathered}
(n+1)^{2} u_{n+1}-\left(11 n^{2}+11 n+3\right) u_{n}-n^{2} u_{n-1}=0, \\
u_{0}^{\prime}=1, \quad u_{1}^{\prime}=3, \quad v_{0}^{\prime}=0, \quad v_{1}^{\prime}=5,
\end{gathered}
$$

and

$$
\begin{gathered}
(n+1)^{3} u_{n+1}-(2 n+1)\left(17 n^{2}+17 n+5\right) u_{n}+n^{3} u_{n}=0 \\
u_{0}^{\prime \prime}=1, \quad u_{1}^{\prime \prime}=5, \quad v_{0}^{\prime \prime}=0, \quad v_{1}^{\prime \prime}=6
\end{gathered}
$$

with the following properties of their solutions:

$$
\lim _{n \rightarrow \infty} \frac{v_{n}^{\prime}}{u_{n}^{\prime}}=\zeta(2), \quad \lim _{n \rightarrow \infty} \frac{v_{n}^{\prime \prime}}{u_{n}^{\prime \prime}}=\zeta(3)
$$


Unexpected inclusions $u_{n}^{\prime}, D_{n}^{2} v_{n}^{\prime} \in \mathbb{Z}$ and $u_{n}^{\prime \prime}, D_{n}^{3} v_{n}^{\prime \prime} \in \mathbb{Z}$, where $D_{n}$ denotes the least common multiple of the numbers $1,2, \ldots, n$ (and $D_{0}=1$ for completeness), together with the prime number theorem $\left(D_{n}^{1 / n} \rightarrow e\right.$ as $\left.n \rightarrow \infty\right)$ and Poincaré's theorem, then yield the following asymptotic behaviour of the linear forms $D_{n}^{2} u_{n}^{\prime} \zeta(2)-D_{n}^{2} v_{n}^{\prime}$ and $D_{n}^{3} u_{n}^{\prime \prime} \zeta(3)-D_{n}^{3} v_{n}^{\prime \prime}$ with integral coefficients:

$$
\begin{aligned}
& \lim _{n \rightarrow \infty}\left|D_{n}^{2} u_{n}^{\prime} \zeta(2)-D_{n}^{2} v_{n}^{\prime}\right|^{1 / n}=e^{2}\left(\frac{\sqrt{5}-1}{2}\right)^{5}<1, \\
& \lim _{n \rightarrow \infty}\left|D_{n}^{3} u_{n}^{\prime \prime} \zeta(3)-D_{n}^{3} v_{n}^{\prime \prime}\right|^{1 / n}=e^{3}(\sqrt{2}-1)^{4}<1,
\end{aligned}
$$

and thus one obtains that both $\zeta(2)$ and $\zeta(3)$ are irrational.

The two following decades after [1] were full of attempts to indicate the total list of the second-order recursions with integral solutions and to show their 'geometric' (i.e., Picard-Fuchs differential equations) origin. We do not pretend to be so heroic in this paper, and we apply quite elementary arguments to get new recurrence equations with 'almost-integral' solutions.

In our general, joint with T. Rivoal, study [9] of arithmetic properties for values of Dirichlet's beta function

$$
\beta(s):=\sum_{l=0}^{\infty} \frac{(-1)^{l}}{(2 l+1)^{s}}
$$

at positive integers $s$, we have discovered a construction of $\mathbb{Q}$-linear forms in 1 and Catalan's constant

$$
G:=\sum_{l=0}^{\infty} \frac{(-1)^{l}}{(2 l+1)^{2}}=\beta(2)
$$

similar to the one considered by Apéry in his proof of the irrationality of $\zeta(2)$. The analogy is far from proving the desired irrationality of $G$, but it allows to indicate the following second-order difference equation

$$
(2 n+1)^{2}(2 n+2)^{2} p(n) u_{n+1}-q(n) u_{n}-(2 n-1)^{2}(2 n)^{2} p(n+1) u_{n-1}=0,
$$

where

$$
\begin{aligned}
& p(n)=20 n^{2}-8 n+1, \\
& q(n)=3520 n^{6}+5632 n^{5}+2064 n^{4}-384 n^{3}-156 n^{2}+16 n+7,
\end{aligned}
$$

with the initial data

$$
u_{0}=1, \quad u_{1}=\frac{7}{4}, \quad v_{0}=0, \quad v_{1}=\frac{13}{8} .
$$

Theorem 1. For each $n=0,1,2, \ldots$, the numbers $u_{n}$ and $v_{n}$ produced by the recursion (2), (4) are positive rationals satisfying the inclusions

$$
2^{4 n+3} D_{n} u_{n} \in \mathbb{Z}, \quad 2^{4 n+3} D_{2 n-1}^{3} v_{n} \in \mathbb{Z},
$$

and the following limit relation holds:

$$
\lim _{n \rightarrow \infty} \frac{v_{n}}{u_{n}}=G .
$$


The positivity and rationality of $u_{n}$ and $v_{n}$ follows immediately from (2)-(4). The characteristic polynomial $\lambda^{2}-11 \lambda-1$ with zeros $((1 \pm \sqrt{5}) / 2)^{5}$ of the difference equation (2) coincides with the corresponding one for Apéry's equation (1). Therefore application of Poincaré's theorem (see also [12], Proposition 2) yields the limit relations

$$
\begin{gathered}
\lim _{n \rightarrow \infty} u_{n}^{1 / n}=\lim _{n \rightarrow \infty} v_{n}^{1 / n}=\left(\frac{1+\sqrt{5}}{2}\right)^{5}=\exp (2.40605912 \ldots), \\
\lim _{n \rightarrow \infty}\left|u_{n} G-v_{n}\right|^{1 / n}=\left|\frac{1-\sqrt{5}}{2}\right|^{5}=\exp (-2.40605912 \ldots),
\end{gathered}
$$

while the inclusions (5) and the prime number theorem imply that the linear forms $2^{4 n+3} D_{2 n-1}^{3}\left(u_{n} G-v_{n}\right)$ with integral coefficients do not tend to 0 as $n \rightarrow \infty$. However, the rational approximations $v_{n} / u_{n}$ to Catalan's constant converge quite rapidly (for instance, $\left.\left|v_{10} / u_{10}-G\right|<10^{-20}\right)$ and one can use the recursion (2), (4) for fast evaluating $G$. Another consequence of Theorem 1 is a new continued-fraction expansion for Catalan's constant. Namely, considering $v_{n} / u_{n}$ as convergents of a continued fraction for $G$ and making the equivalent transform of the fraction ([6], Theorems 2.2 and 2.6) we arrive at

Theorem 2. The following expansion holds:

$$
G=\frac{13 / 2}{\mid q(0)}+\frac{1^{4} \cdot 2^{4} \cdot p(0) p(2) \mid}{q(1)}+\cdots+\frac{(2 n-1)^{4}(2 n)^{4} p(n-1) p(n+1)}{q(n)}+\cdots,
$$

where the polynomials $p(n)$ and $q(n)$ are given in (3).

The multiple-integral representation for the linear forms $u_{n} G-v_{n}$ similar to those obtained by F. Beukers in [4], formula (5), for the linear forms $u_{n}^{\prime} \zeta(2)-v_{n}^{\prime}$ is given by

Theorem 3. For each $n=0,1,2, \ldots$, the identity

$$
u_{n} G-v_{n}=\frac{(-1)^{n}}{4} \int_{0}^{1} \int_{0}^{1} \frac{x^{n-1 / 2}(1-x)^{n} y^{n}(1-y)^{n-1 / 2}}{(1-x y)^{n+1}} \mathrm{~d} x \mathrm{~d} y
$$

holds.

\section{Difference equation for Catalan's constant}

Consider the rational function

$$
R_{n}(t):=n !(2 t+n+1) \frac{t(t-1) \cdots(t-n+1) \cdot(t+n+1) \cdots(t+2 n)}{\left(\left(t+\frac{1}{2}\right)\left(t+\frac{3}{2}\right) \cdots\left(t+n+\frac{1}{2}\right)\right)^{3}}
$$


and the corresponding (very-well-poised) hypergeometric series

$$
\begin{aligned}
& F_{n}:=\sum_{t=0}^{\infty}(-1)^{t} R_{n}(t) \\
& =(-1)^{n} n ! \frac{\Gamma(3 n+2) \Gamma\left(n+\frac{1}{2}\right)^{3} \Gamma(n+1)}{\Gamma\left(2 n+\frac{3}{2}\right)^{3} \Gamma(2 n+1)}
\end{aligned}
$$

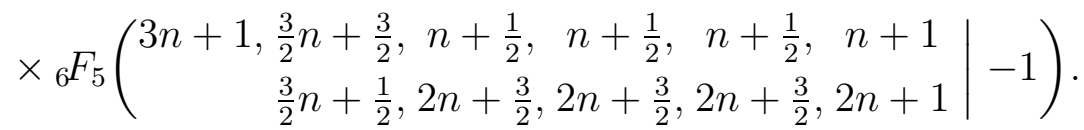

Lemma 1. The following equality holds:

$$
F_{n}=U_{n} \beta(3)+U_{n}^{\prime} \beta(2)+U_{n}^{\prime \prime} \beta(1)-V_{n},
$$

where $U_{n}, D_{n} U_{n}^{\prime}, D_{n}^{2} U_{n}^{\prime \prime}, D_{2 n-1}^{3} V_{n} \in 2^{-4 n} \mathbb{Z}$.

Proof. We start with mentioning that

$$
P_{n}^{(1)}(t):=\frac{t(t-1) \cdots(t-n+1)}{n !} \quad \text { and } \quad P_{n}^{(2)}(t):=\frac{(t+n+1) \cdots(t+2 n)}{n !}
$$

are integral-valued polynomials and, as it is known (see, e.g., [13], Lemma 7),

$$
2^{2 n} \cdot P_{n}\left(-k-\frac{1}{2}\right) \in \mathbb{Z} \quad \text { for } \quad k \in \mathbb{Z}
$$

and, moreover,

$$
\left.2^{2 n} D_{n}^{j} \cdot \frac{1}{j !} \frac{\mathrm{d}^{j} P_{n}(t)}{\mathrm{d} t^{j}}\right|_{t=-k-1 / 2} \in \mathbb{Z} \quad \text { for } \quad k \in \mathbb{Z} \quad \text { and } \quad j=1,2, \ldots,
$$

where $P_{n}(t)$ is any of the polynomials (10).

The rational function

$$
Q_{n}(t):=\frac{n !}{\left(t+\frac{1}{2}\right)\left(t+\frac{3}{2}\right) \cdots\left(t+n+\frac{1}{2}\right)}
$$

has also 'nice' arithmetic properties. Namely,

$$
a_{k}:=\left.Q_{n}(t)\left(t+k+\frac{1}{2}\right)\right|_{t=-k-1 / 2}= \begin{cases}(-1)^{k}\left(\begin{array}{l}
n \\
k
\end{array}\right) \in \mathbb{Z} & \text { if } k=0,1, \ldots, n, \\
0 & \text { for other } k \in \mathbb{Z}\end{cases}
$$

that allow to write the following partial-fraction expansion:

$$
Q_{n}(t)=\sum_{l=0}^{n} \frac{a_{l}}{t+l+\frac{1}{2}}
$$


Hence, for $j=1,2, \ldots$ we obtain

$$
\begin{aligned}
\left.\frac{D_{n}^{j}}{j !} \frac{\mathrm{d}^{j}}{\mathrm{~d} t^{j}}\left(Q_{n}(t)\left(t+k+\frac{1}{2}\right)\right)\right|_{t=-k-1 / 2} & =\left.\frac{D_{n}^{j}}{j !} \frac{\mathrm{d}^{j}}{\mathrm{~d} t^{j}} \sum_{l=0}^{n} a_{l}\left(1-\frac{l-k}{t+l+\frac{1}{2}}\right)\right|_{t=-k-1 / 2} \\
& =(-1)^{j-1} D_{n}^{j} \sum_{\substack{l=0 \\
l \neq k}}^{n} \frac{1}{(l-k)^{j}} \in \mathbb{Z} .
\end{aligned}
$$

Therefore the inclusions (11)-(14) and the Leibniz rule for differentiating a product imply that the numbers

$$
\begin{aligned}
A_{j k} & =A_{j k}(n):=\left.\frac{1}{j !} \frac{\mathrm{d}^{j}}{\mathrm{~d} t^{j}}\left(R_{n}(t)\left(t+k+\frac{1}{2}\right)^{3}\right)\right|_{t=-k-1 / 2} \\
& =\left.\frac{1}{j !} \frac{\mathrm{d}^{j}}{\mathrm{~d} t^{j}}\left((2 t+n+1) \cdot P_{n}^{(1)}(t) \cdot P_{n}^{(2)}(t) \cdot\left(Q_{n}(t)\left(t+k+\frac{1}{2}\right)\right)^{3}\right)\right|_{t=-k-1 / 2}
\end{aligned}
$$

satisfy the inclusions

$$
2^{4 n} D_{n}^{j} \cdot A_{j k}(n) \in \mathbb{Z} \quad \text { for } \quad k=0,1, \ldots, n \quad \text { and } \quad j=0,1,2, \ldots
$$

Mention now that the numbers (15) are coefficients in the partial-fraction expansion of the rational function $(7)$,

$$
R_{n}(t)=\sum_{j=0}^{2} \sum_{k=0}^{n} \frac{A_{j k}}{\left(t+k+\frac{1}{2}\right)^{3-j}}
$$

Substituting this expansion into the definition (8) of the quantity $F_{n}$ we obtain the desired representaion (9):

$$
\begin{aligned}
F_{n} & =\sum_{j=0}^{2} \sum_{k=0}^{n}(-1)^{k} A_{j k} \sum_{t=0}^{\infty} \frac{(-1)^{t+k}}{\left(t+k+\frac{1}{2}\right)^{3-j}} \\
& =\sum_{j=0}^{2} \sum_{k=0}^{n}(-1)^{k} A_{j k}\left(\sum_{l=0}^{\infty}-\sum_{l=0}^{k-1}\right) \frac{(-1)^{l}}{\left(l+\frac{1}{2}\right)^{3-j}} \\
& =U_{n} \beta(3)+U_{n}^{\prime} \beta(2)+U_{n}^{\prime \prime} \beta(1)-V_{n},
\end{aligned}
$$

where

$$
\begin{gathered}
U_{n}=2^{3} \sum_{k=0}^{n}(-1)^{k} A_{0 k}(n), \quad U_{n}^{\prime}=2^{2} \sum_{k=0}^{n}(-1)^{k} A_{1 k}(n), \quad U_{n}^{\prime \prime}=2 \sum_{k=0}^{n}(-1)^{k} A_{2 k}(n), \\
V_{n}=\sum_{j=0}^{2} 2^{3-j} \sum_{k=0}^{n}(-1)^{k} A_{j k}(n) \sum_{l=0}^{k-1} \frac{(-1)^{l}}{(2 l+1)^{3-j}} .
\end{gathered}
$$


Finally, using the inclusions (16) and

$$
D_{2 n-1}^{3-j} \sum_{l=0}^{k-1} \frac{(-1)^{l}}{(2 l+1)^{3-j}} \in \mathbb{Z} \quad \text { for } \quad k=0,1, \ldots, n \quad \text { and } \quad j=0,1,2,
$$

we deduce that $U_{n}, D_{n} U_{n}^{\prime}, D_{n}^{2} U_{n}^{\prime \prime}, D_{2 n-1}^{3} V_{n} \in 2^{-4 n} \mathbb{Z}$ as required.

Using Zeilberger's algorithm of creative telescoping ([7], Section 6) for the rational function (7), we obtain the certificate $S_{n}(t):=s_{n}(t) R_{n}(t)$, where

$$
\begin{aligned}
& s_{n}(t):=\frac{1}{2(2 t+n+1)(t+2 n-1)(t+2 n)} \cdot\left(8 n(2 n-1)^{2}\left(20 n^{2}+32 n+13\right) t^{4}\right. \\
& \quad+2\left(5440 n^{6}+7104 n^{5}+912 n^{4}-1088 n^{3}+76 n^{2}+68 n+7\right) t^{3} \\
& \quad+\left(44800 n^{7}+65600 n^{6}+17568 n^{5}-7056 n^{4}-1088 n^{3}+372 n^{2}+146 n-1\right) t^{2} \\
& \quad+(2 n+1)\left(34880 n^{7}+39328 n^{6}-2176 n^{5}-8416 n^{4}+964 n^{3}+154 n^{2}+58 n-13\right) t \\
& \left.\quad+n(2 n-1)(2 n+1)^{2}\left(4720 n^{5}+6192 n^{4}+816 n^{3}-864 n^{2}+69 n+13\right)\right)
\end{aligned}
$$

satisfying the following property.

Lemma 2. For each $n=1,2, \ldots$, we have the identity

$$
\begin{aligned}
& (2 n+1)^{2}(2 n+2)^{2} p(n) R_{n+1}(t)-q(n) R_{n}(t)-(2 n-1)^{2}(2 n)^{2} p(n+1) R_{n-1}(t) \\
& \quad=-S_{n}(t+1)-S_{n}(t),
\end{aligned}
$$

where the polynomials $p(n)$ and $q(n)$ are given in (3).

Proof. Divide both sides of $(21)$ by $R_{n}(t)$ and verify the identity

$$
\begin{gathered}
(2 n+1)^{2}(2 n+2)^{2} p(n) \cdot(n+1) \frac{(2 t+n+2)(t-n)(t+2 n+1)(t+2 n+2)}{(2 t+n+1)(t+n+1)\left(t+n+\frac{3}{2}\right)^{3}}-q(n) \\
\quad-(2 n-1)^{2}(2 n)^{2} p(n+1) \cdot \frac{(2 t+n)(t+n)\left(t+n+\frac{1}{2}\right)^{3}}{n(2 t+n+1)(t-n+1)(t+2 n-1)(t+2 n)} \\
=-s_{n}(t+1) \frac{(2 t+n+3)\left(t+\frac{1}{2}\right)^{3}(t+1)(t+2 n+1)}{(2 t+n+1)(t-n+1)(t+n+1)\left(t+n+\frac{3}{2}\right)^{3}}-s_{n}(t),
\end{gathered}
$$

where $s_{n}(t)$ is given in $(20)$.

Lemma 3. The quantity (8) satisfies the difference equation (2) for $n=1,2, \ldots$.

Proof. Multiplying both sides of the equality $(21)$ by $(-1)^{t}$ and summing the result over $t=0,1,2, \ldots$ we obtain

$$
(2 n+1)^{2}(2 n+2)^{2} p(n) F_{n+1}-b(n) F_{n}-(2 n-1)^{2}(2 n)^{2} p(n+1) F_{n-1}=-S_{n}(0) .
$$

It remains to note that, for $n \geq 1$, both functions $R_{n}(t)$ and $S_{n}(t)=s_{n}(t) R_{n}(t)$ have zero at $t=0$. Thus $S_{n}(0)=0$ for $n=1,2, \ldots$ and we obtain the desired recurrence (2) for the quantity (8). 
Lemma 4. The coefficients $U_{n}, U_{n}^{\prime}, U_{n}^{\prime \prime}, V_{n}$ in the representation (9) satisfy the difference equation (2) for $n=1,2, \ldots$.

Proof. We can write down the partial-fraction expansion (17) in the form

$$
R_{n}(t)=\sum_{j=1}^{4} \sum_{k=-\infty}^{+\infty} \frac{A_{j k}(n)}{\left(t+k+\frac{1}{2}\right)^{3-j}}
$$

where the formulae (15) remain true for all $k \in \mathbb{Z}$ (not for $k=0,1, \ldots, n$ only). Now, multiply both sides of $(21)$ by $(-1)^{k}\left(t+k+\frac{1}{2}\right)^{3}$, take the $j$ th derivative, where $j=0,1,2$, substitute $t=-k-\frac{1}{2}$ in the result, and sum over all integers $k$; this procedure implies that the numbers

$$
U_{n}=8 \sum_{k=-\infty}^{+\infty}(-1)^{k} A_{0 k}(n), \quad U_{n}^{\prime}=4 \sum_{k=-\infty}^{+\infty}(-1)^{k} A_{1 k}(n), \quad U_{n}^{\prime \prime}=2 \sum_{k=-\infty}^{+\infty}(-1)^{k} A_{2 k}(n)
$$

(cf. (18)) satisfy the difference equation (2). Finally, the sequence

$$
V_{n}=U_{n} \beta(3)+U_{n}^{\prime} \beta(2)+U_{n}^{\prime \prime} \beta(1)-F_{n}
$$

also satisfies the recursion (2) by Lemma 3 and the above.

Since

$$
R_{0}(t)=\frac{2}{\left(t+\frac{1}{2}\right)^{2}}, \quad R_{1}(t)=-\frac{3 / 4}{\left(t+\frac{1}{2}\right)^{3}}-\frac{3 / 4}{\left(t+\frac{3}{2}\right)^{3}}+\frac{7 / 4}{\left(t+\frac{1}{2}\right)^{2}}-\frac{7 / 4}{\left(t+\frac{3}{2}\right)^{2}},
$$

in accordance with (18), (19) we obtain

$$
U_{0}^{\prime}=8, \quad U_{0}=U_{0}^{\prime \prime}=V_{0}=0, \quad \text { and } \quad U_{1}^{\prime}=14, \quad V_{1}=13, \quad U_{1}=U_{1}^{\prime \prime}=0,
$$

hence as a consequence of Lemma 4 we deduce that $U_{n}=U_{n}^{\prime \prime}=0$ for $n=0,1,2, \ldots$.

Lemma 5. The following equality holds:

$$
F_{n}=U_{n}^{\prime} G-V_{n}
$$

where $2^{4 n} D_{n} U_{n}^{\prime} \in \mathbb{Z}$ and $2^{4 n} D_{2 n-1}^{3} V_{n} \in \mathbb{Z}$.

The sequences $u_{n}:=U_{n}^{\prime} / 8$ and $v_{n}:=V_{n} / 8$ satisfy the difference equation (2) and initial conditions (4); the fact that $F_{n} \neq 0$ and $\left|F_{n}\right| \rightarrow 0$ as $n \rightarrow \infty$ follows from Theorem 3 and asymptotics of the multiple integral (6) proved in [4]. This completes the proof of Theorem 1. 


\section{Connection with ${ }_{3} F_{2}$-hypergeometric series}

The corresponding very-well-poised hypergeometric series (8) at $z=-1$ can be reduced to a simpler series with the help of Whipple's transform ([2], Section 4.4, formula (2)):

$$
\begin{aligned}
{ }_{3} F_{2}\left(\begin{array}{c}
1+a-b-c, d, e \\
1+a-b, 1+a-c
\end{array}\right. & 1)=\frac{\Gamma(1+a) \Gamma(1+a-d-e)}{\Gamma(1+a-d) \Gamma(1+a-e)} \\
& \times{ }_{6} F_{5}\left(\begin{array}{r}
a, 1+\frac{1}{2} a, \quad b, \quad c, \quad d, \\
\frac{1}{2} a, \quad 1+a-b, 1+a-c, 1+a-d, 1+a-e
\end{array} \mid-1\right)
\end{aligned}
$$

if $\operatorname{Re}(1+a-d-e)>0$. Namely, in the case $a=3 n+1, b=c=d=n+\frac{1}{2}$, and $e=n+1$, we obtain

$$
F_{n}=U_{n}^{\prime} G-V_{n}=(-1)^{n} \cdot 2 \int_{0}^{1} \int_{0}^{1} \frac{x^{n-1 / 2}(1-x)^{n} y^{n}(1-y)^{n-1 / 2}}{(1-x y)^{n+1}} \mathrm{~d} x \mathrm{~d} y,
$$

where the Euler-type integral representation for the ${ }_{3} F_{2}$-series can be derived as in [10], Section 4.1, and [3], the proof of Lemma 2.

This completes the proof of Theorem 3.

\section{Concluding remarks}

The conclusion (5) of Theorem 1 is far from being precise; in fact, using (2), (4) one gets experimentally (up to $n=1000$, say) the stronger inclusions ${ }^{1}$

$$
2^{4 n} u_{n} \in \mathbb{Z}, \quad 2^{4 n} D_{2 n-1}^{2} v_{n} \in \mathbb{Z} .
$$

Unfortunately, they also give no chance to prove that Catalan's constant is irrational since linear forms $2^{4 n} D_{2 n-1}^{2}\left(u_{n} G-v_{n}\right)$ do not tend to 0 as $n \rightarrow \infty$.

In the same vein, using another very-well-poised series of hypergeometric type

$$
\begin{aligned}
\widetilde{F}_{n} & :=\frac{(-1)^{n+1}}{6} \sum_{t=1}^{\infty} \frac{\mathrm{d}}{\mathrm{d} t}\left((2 t+n) \frac{((t-1) \cdots(t-n))^{2} \cdot((t+n+1) \cdots(t+2 n))^{2}}{(t(t+1) \cdots(t+n))^{4}}\right) \\
& =\widetilde{u}_{n} \zeta(4)-\widetilde{v}_{n}
\end{aligned}
$$

and the arguments of Section 2, we deduce the difference equation

$$
(n+1)^{5} u_{n+1}-r(n) u_{n}-3 n^{3}(3 n-1)(3 n+1) u_{n-1}=0,
$$

where

$$
\begin{aligned}
r(n) & =3(2 n+1)\left(3 n^{2}+3 n+1\right)\left(15 n^{2}+15 n+4\right) \\
& =270 n^{5}+675 n^{4}+702 n^{3}+378 n^{2}+105 n+12,
\end{aligned}
$$

\footnotetext{
${ }^{1} \mathrm{~A}$ slightly weakened form of the inclusions is proved in [15].
} 
with the initial data

$$
\widetilde{u}_{0}=1, \quad \widetilde{u}_{1}=12, \quad \widetilde{v}_{0}=0, \quad \widetilde{v}_{1}=13
$$

for its two independent solutions $\widetilde{u}_{n}$ and $\widetilde{v}_{n}$,

Theorem 4 ([14]). For each $n=0,1,2, \ldots$, the numbers $\widetilde{u}_{n}$ and $\widetilde{v}_{n}$ are positive rationals satisfying the inclusions

$$
6 D_{n} \widetilde{u}_{n} \in \mathbb{Z}, \quad 6 D_{n}^{5} \widetilde{v}_{n} \in \mathbb{Z},
$$

and the following limit relation holds:

$$
\lim _{n \rightarrow \infty} \frac{\widetilde{v}_{n}}{\widetilde{u}_{n}}=\frac{\pi^{4}}{90}=\zeta(4)=\sum_{n=1}^{\infty} \frac{1}{n^{4}} .
$$

Remark. During the preparation of this article, we have known that the difference equation (22), in slightly different normalization, and the limit relation (25) without the inclusions (24) had been stated independently by H. Cohen and G. Rhin [5] using Apéry's 'accélération de la convergence' approach, and by V. N. Sorokin [11] by means of certain explicit Hermite-Padé-type approximations. We underline that our approach differs from that of [5] and [11].

Application of Poincaré's theorem yields the asymptotic relations

$$
\lim _{n \rightarrow \infty}\left|\widetilde{u}_{n}\right|^{1 / n}=\lim _{n \rightarrow \infty}\left|\widetilde{v}_{n}\right|^{1 / n}=(3+2 \sqrt{3})^{3}=\exp (5.59879212 \ldots)
$$

and

$$
\lim _{n \rightarrow \infty}\left|\widetilde{u}_{n} \zeta(4)-\widetilde{v}_{n}\right|^{1 / n}=|3-2 \sqrt{3}|^{3}=\exp (-2.30295525 \ldots),
$$

since the characteristic polynomial $\lambda^{2}-270 \lambda-27$ of the equation (22) has zeros $135 \pm$ $78 \sqrt{3}=(3 \pm 2 \sqrt{3})^{3}$. Thus, we can consider $\widetilde{v}_{n} / \widetilde{u}_{n}$ as convergents of a continued fraction for $\zeta(4)$ and making the equivalent transform of the fraction we obtain

Theorem 5. The following expansion holds:

$$
\zeta(4)=\frac{13}{r(0)}+\frac{1^{7} \cdot 2 \cdot 3 \cdot 4}{r(1)}+\frac{2^{7} \cdot 5 \cdot 6 \cdot 7}{r r(2)}+\cdots+\frac{n^{7}(3 n-1)(3 n)(3 n+1)}{r(n)}+\cdots,
$$

where the polynomial $r(n)$ is given in (23).

The mystery of the $\zeta(4)$-case consists in the fact that experimental calculations give us the better inclusions

$$
\widetilde{u}_{n} \in \mathbb{Z}, \quad D_{n}^{4} \widetilde{v}_{n} \in \mathbb{Z}
$$

(cf. (24)); unfortunately, the linear forms $D_{n}^{4}\left(\widetilde{u}_{n} \zeta(4)-\widetilde{v}_{n}\right)$ do not tend to 0 as $n \rightarrow \infty$. 


\section{References}

[1] R. Apéry, "Irrationalité de $\zeta(2)$ et $\zeta(3) "$, Astérisque 61 (1979), 11-13.

[2] W. N. Bailey, Generalized hypergeometric series, Cambridge Math. Tracts 32, Cambridge Univ. Press, Cambridge, 1935; 2nd reprinted edition, Stechert-Hafner, New York-London, 1964.

[3] K. Ball and T. Rivoal, "Irrationalité d'une infinité de valeurs de la fonction zêta aux entiers impairs", Invent. Math. 146:1 (2001), 193-207.

[4] F. Beukers, "A note on the irrationality of $\zeta(2)$ and $\zeta(3)$ ", Bull. London Math. Soc. 11:3 (1979), 268-272.

[5] H. Cohen, "Accélération de la convergence de certaines récurrences linéaires", Séminaire de Théorie des nombres de Bordeaux (Année 1980-81), exposé 16, 2 pages.

[6] W. B. Jones and W. J. Thron, Continued fractions. Analytic theory and applications, Encyclopaedia Math. Appl. Section: Analysis 11, Addison-Wesley, London, 1980.

[7] M. Petkovšek, H. S. Wilf, and D. Zeilberger, $A=B$, A. K. Peters, Ltd., Wellesley, MA, 1997.

[8] A. van der Poorten, "A proof that Euler missed... Apéry's proof of the irrationality of $\zeta(3)$ ", An informal report, Math. Intelligencer 1:4 (1978/79), 195-203.

[9] T. Rivoal and W. Zudilin, "Diophantine properties of numbers related to Catalan's constant", Math. Annalen (2003), to appear; Prépubl. de l'Institut de Math. de Jussieu, no. 315 (January 2002), 17 pages.

[10] L. J. Slater, Generalized hypergeometric functions, Cambridge Univ. Press, Cambridge, 1966.

[11] V. N. Sorokin, "One algorithm for fast calculation of $\pi^{4}$ ", Preprint (April 2002), Russian Academy of Sciences, M. V. Keldysh Institute for Applied Mathematics, Moscow, 2002, 59 pages; available at http://www.wis.kuleuven.ac.be/applied/intas/Art5.pdf.

[12] V. V. Zudilin [W. Zudilin], "Difference equations and the irrationality measure of numbers", Collection of papers: Analytic number theory and applications, Trudy Mat. Inst. Steklov [Proc. Steklov Inst. Math.] 218 (1997), 165-178.

[13] V. V. Zudilin [W. Zudilin], "Cancellation of factorials", Mat. Sb. [Russian Acad. Sci. Sb. Math.] 192:8 (2001), 95-122.

[14] W. Zudilin, "Well-poised hypergeometric service for diophantine problems of zeta values", Actes des 12èmes rencontres arithmétiques de Caen (June 29-30, 2001), J. Théorie Nombres Bordeaux, to appear.

[15] W. Zudilin, "A few remarks on linear forms involving Catalan's constant", Chebyshevskiu Sbornik (Tula State Pedagogical University) 3:2 (4) (2002), 60-70; English transl., E-print http://arXiv.org/math.NT/0210423 (October 2002). 